\title{
Endothelialization of Acellular Porcine ECM with Chemical Modification
}

\author{
Yao Wang, Tomer Bronshtein, Udi Sarig, Freddy Yin Chiang Boey, Subbu S. Venkatraman, and
} Marcelle Machluf

\begin{abstract}
Vascularization remains a critical requirement for the long term survival of engineered tissue constructs, especially thick ones. Such thick constructs for cardiac tissue engineering has been reported by our group and others based on decellularized porcine cardiac extracellular matrix (pcECM) that has been shown to resemble the native tissue both structurally and chemically. The network of inherent vasculature, which was largely retained within our PcECM, can be used as primers for re-endothelialization and neo-vascularization with regenerative cells. Endothelial cells alone, seeded onto the ECM, not only attached and survived but also rearranged into typical confluent monolayer with self-alignment. Sequential co-cultures of human umbilical vein endothelial cells (HUVEC) and mesenchymal stem cells (MSC) were shown to support the growth of both lineages on the surface and in the vasculature of reseeded pcECM. After ECM treatment with gelatin or fibronectin, cell proliferation increased significantly for both MSCs and HUVECs. Preliminary results showed that future efforts combining co-culture, treated scaffolds and dynamic culture environment may result in re-endothelialization leading to functional blood vessels in thick engineered tissue for partial cardiac replacement therapy.
\end{abstract}

Keywords-Cardiovascular, tissue regeneration, tissue engineering, vascularization, extracellular matrix

\section{INTRODUCTION}

Myocardial infarction (MI), commonly referred to as a heart attack, is mostly caused by blockage of the major blood supply to the cardiac tissue, leading to ischemia and cell death. As adult cardiomyocytes are terminally differentiated, once the tissue is injured, the human heart cannot repair itself and a scar tissue is formed. The excess mechanical load on the damaged tissue, which induces ventricular remodeling, will eventually lead to congestive heart failure [1]. While heart transplantation remains the treatment of choice for end-stage heart diseases, donor shortage and immune rejection still limit its applications [2]. Hence, alternative approaches have been suggested for heart regeneration utilizing cardiac patches made from biocompatible materials, seeded with expandable and functional human cells, and

Manuscript received August 1, 2012; revised September 10, 2012. This work was supported in part by the Singapore National Research Foundation.

Y. Wang is with the Nanyang Technological University, Singapore, Nanyang Avenue, 639798 Singapore and Technion - Israel Institute of Technology, Haifa, 32000 Israel (e-mail: WANG0580@ntu.edu.sg).

T. Bronshtein, U. Sarig, F. Y. C. Boey, and S. S. Venkatraman are with the Nanyang Technological University, Singapore, Nanyang Avenue, 639798 Singapore (e-mail: TBRONSHTEIN@ntu.edu.sg, ESarig@NTU.edu.sg,MYCBOEY@ntu.edu.sg, ASSUBBU@ntu.edu.sg).

M. Machluf is with the Technion - Israel Institute of Technology, Haifa, 32000 Israel (e-mail: machlufm@tx.technion.ac.il). grown in supportive environments [1], [3].

The successful regeneration of thick tissue like cardiac left ventricles has been greatly hampered by the lack of vascularization [4], [5]. Cardiac tissue possesses a complicated and hierarchical network of blood vessels. From veins and arteries to capillaries, regardless of their sizes and locations, every single blood vessel plays an essential role in transporting nutrients and oxygen throughout the cardiac tissue. In the absence of vasculature, regenerated tissue has a critical diffusion thickness, greater than which, diffusion of oxygen and nutrients will not be sufficient to support the needs of newly formed tissue. Work performed by Radisic et al. indicated that without functional vascularization, the mass transport can only reach as far as $100 \mu \mathrm{m}$ into the tissue. Perfusion bioreactors were shown to effectively increase the cell penetration depth to about $200 \mu \mathrm{m}$ [6]. Nonetheless, once the regenerated tissue is transplanted to the host body, cell survival will be largely compromised without functional blood vessel network.

Acellular cardiac extracellular matrix (ECM), which largely possesses chemical and mechanical properties desirable to mimic environment in native cardiac tissue, has been suggested as a suitable scaffold material for cardiovascular tissue engineering [7], [8]. An effective process to isolate acellular ECM from porcine myocardium tissue, was reported by us and was shown to maintain the mechanical and biochemical properties and the major ECM components as well as support the long-term survival of cardiomyocytes and mesenchymal stem cells (MSC) [7]. Moreover, thick acellular ECM patch derived from porcine left ventricle was shown to retain the major structure of its inherent vasculature [9] that can be used ex- or in vivo as conduits to feed the tissue bulk, thus overcome the diffusion barrier limiting survival of cells more than $100 \mu \mathrm{m}$ away from the nearest blood vessel [10]. Yet, the re-endothelialization of these conduits remains a critical problem requiring optimized conditions to support the attachment, survival, and proliferation of endothelial cells, which might facilitate future functional angiogenesis upon transplantation.

Angiogenesis is commonly achieved by sprouting of newly recruited cells from existing blood vessels with the help of mural cells [11]. Mural cells, including vascular smooth muscle cells and pericytes, together with endothelial cells, possess the potential of recruiting cells from the surrounding tissue in vivo [12]. MSCs can be differentiated into both endothelial and mural cells in vitro and in vivo [13]. Our preliminary studies demonstrated that endothelial cells seeded into the vasculature of the ECM were only shown to survive and reach a stabilized phase over time without 
proliferation, which is necessary for the formation of functional blood vessels. As such, relying on the potential of co-cultures and different ECM components to influence the endothelial cells, we have tested the effects of co-culturing and several ECM modifications on the growth and rearrangement of human umbilical cord endothelial cells (HUVEC). Our studies revealed that chemical treatment for scaffold material and co-culturing can both significantly increase the proliferation of endothelial cells. The suitability of acellular porcine cardiac ECM as candidate scaffold for vascularization and angiogenesis for cardiac tissue regeneration was also attested.

\section{MATERIALS AND METHODS}

\section{A. Cell Culture}

Human bone marrow derived MSC and HUVEC were both purchased from Lonza (Basel, Switzerland). MSCs were cultured in Minimum Essential Medium Eagle, alpha modification ( $\alpha \mathrm{MEM}$, Sigma $^{\mathrm{TM}}$, St. Louis, MO) supplemented with 10\% fetal bovine serum (Gibco, Langley, OK), 1\% L-Glutamine (Gibco), 5 ng/ml bFGF (Invitrogen, Carlsbad, CA), and 1\% Antibiotic-Antimycotic (Gibco). HUVECs were cultured in EGM-2 complete medium (Lonza) supplemented with 1\% Antibiotic-Antimycotic (Gibco).

\section{B. Decellularization of Porcine Cardiac ECM}

Acellular porcine cardiac ECM was produced according to our previously reported decellularization process [7], [9]. Briefly, the left ventricles from healthy female pigs were excised into $70 \mathrm{~mm}(\mathrm{w})$ x $90 \mathrm{~mm}(\mathrm{l}) \times 10 \mathrm{~mm}$ (t) tissue slabs containing left anterior descending coronary artery and its adjacent veins. The tissue slabs were then decellularized with washing cycles including alternating hyper/hypo tonic solutions: $1.1 \%$ and $0.7 \%(\mathrm{w} / \mathrm{v}) \mathrm{NaCl}$ in double distilled water (DDW) respectively, $0.05 \%(\mathrm{w} / \mathrm{v})$ trypsin $\left(\mathrm{Sigma}^{\mathrm{TM}}\right)$ supplemented with $0.02 \%(\mathrm{w} / \mathrm{v})$ EDTA $\left(\operatorname{Sigma}^{\mathrm{TM}}\right)$, and $1 \%$ (v/v) Triton-X-100 (BioLab Ltd., Jerusalem, Israel) in phosphate buffered saline (PBS) supplemented with $1 \%(\mathrm{v} / \mathrm{v})$ ammonium hydroxide (BioLab Ltd.). To enhance the efficiency of washing, the tissue slabs were sonicated for 30 minutes twice a day in an ultrasound bath (Jeio-tech, Seoul, Korea).

\section{ECM Preparation}

After decellularization process, ECM slab was cut into cylindrical pieces with bottom area of about $0.3-0.5 \mathrm{~cm}^{2}$. ECM slices were then washed with $70 \%$ ethanol, followed by PBS and EGM-2 washes. For chemical modification, 4 $\mathrm{mg} / \mathrm{ml}$ gelatin $\left(\operatorname{Sigma}^{\mathrm{TM}}\right), 10 \mu \mathrm{g} / \mathrm{ml}$ fibronectin $\left(\operatorname{Sigma}^{\mathrm{TM}}\right)$, and $100 \mu \mathrm{g} / \mathrm{ml}$ laminin (Sigma ${ }^{\mathrm{TM}}$ ) coating solutions were prepared by dissolving protein powders in PBS. ECM slices were immersed in each of the coating solutions for 24 hours. Un-coated ECM served as control.

\section{Cell Seeding}

Prior to seeding, ECM slices were individually placed into 96-well low binding plate (NUNCTM, Roskilde, Denmark). For attachment test of HUVEC on ECM, 200,000 cells were harvested, re-suspended in 40 $\mu$ EGM-2 medium, and seeded on top of ECM slices with a pipette. The samples were incubated at $37^{\circ} \mathrm{C}$ and $5 \% \mathrm{CO}_{2}$ for $0,45,90,135$, and 180 minutes before brief washing with PBS and fixation with $2 \%$ PFA for histological studies.

For simultaneous co-culture, 150,000 MSCs and 150,000 of HUVECs were seeded together in a mixed suspension on each ECM slice. For sequential co-culture, HUVECs were seeded 7 days after MSCs. For single culture, 300,000 HUVECs or 300,000 MSCs were seeded separately. Seeded ECM slices were cultured in 24-well low binding plates (NUNCTM) in EGM-2 for 21 days. Medium was replenished every second day.

\section{E. Alamar Blue ${ }^{\mathrm{TM}}$ Assay}

Re-seeded ECM slices were incubated in EGM-2 with 10\% AlamarBlue $^{\mathrm{TM}}$ reagent (Invitrogen) for 5 hours. The fluorescence intensity was measured with Varioskan Flash spectral scanning multimode reader (Thermo Fisher Scientific, Waltham, MA) according to the manufacturer's instructions. ECM with no cells served as control. The viability of the cells was calculated from the AlamarBlue ${ }^{\mathrm{TM}}$ readings which were taken every second day.

\section{F. Scanning Electron Microscopy}

ECM samples seeded with cells were gently washed with PBS and fixed in 2\% PFA overnight. Samples were then washed with PBS three times and dehydrated with ethanol in ascending concentration from $30 \%$ to $100 \%$, and air dried. The dehydrated samples were sputtered with gold molecules and mounted on JSM-6360 scanning electron microscope for imaging (JEOL, Tokyo, Japan).

\section{G. Cell labeling with DII}

Cells were harvested and incubated with $12.5 \mu \mathrm{M}$ non-specific membrane labeling dye DiI (Invitrogen) for 30 minutes at $37^{\circ} \mathrm{C}$, followed by washing three times with PBS. To remove excess dye, cells were then plated and cultured overnight prior to seeding on ECM.

\section{H. Histology Study}

The attachment of HUVECs on ECM was assessed using H\&E staining on cross-sections of samples after cryosectioning, performed by Histopathology Unit, IMCB (Singapore).

\section{Statistical Analysis}

Averaged results are presented as mean \pm SE of $n \geq 3$. Two-tailed t-test was used to determine the difference between means. Analysis of variance (ANOVA) was used to test the statistical significance of differences among groups. Statistically significant difference is defined for $\mathrm{p}<0.05$.

\section{RESULTS}

\section{A. HUVEC Attachment on ECM}

The attachment of HUVECs on ECM slices was assessed by both AlamarBlue ${ }^{\mathrm{TM}}$ assay and H\&E staining. The percentage of attachment from AlamarBlue ${ }^{\mathrm{TM}}$ result was calculated against total initial seeding number and a calibration curve. Highest degree of attachment (about 30\%) was observed 45 to 90 minutes after seeding. Only $20 \%$ attachment was achieved immediately after seeding or with 180 minutes incubation. AlamarBlue ${ }^{\mathrm{TM}}$ reading after 135 
minutes incubation time fell in the middle showing $26 \%$ attachment rate (Fig. 1).

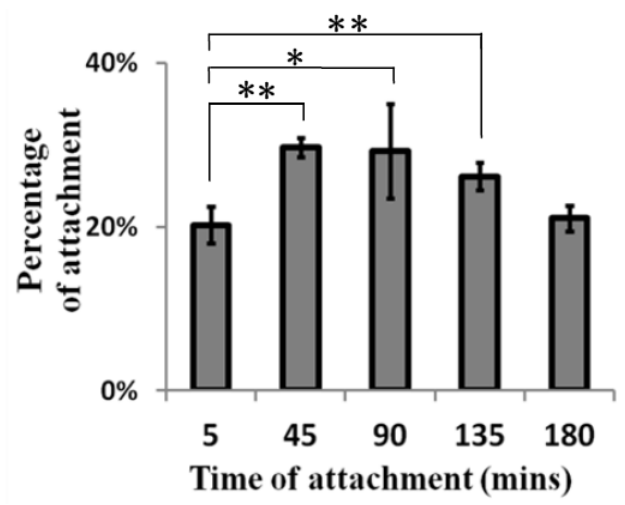

Fig. 1. HUVEC attachment on ECM slices from AlamarBlue ${ }^{\mathrm{TM}}$ assay. Best attachment efficiency of about $30 \%$ was achieved after static incubation of 45 to 90 minutes $(* p<0.05, * * p<0.005)$.

Histological images from $H \& E$ staining proved the absence of cells in decellularized ECM (Fig. 2A) in contrast to native tissue (Fig. 2B). Images taken from re-cellularized ECM samples after different attachment time appeared to be consistent with the AlamarBlue ${ }^{\mathrm{TM}}$ assay (Fig. 3). Cross-sectional images were taken randomly from more than 3 regions of interests (ROI) for each sample slice. Very few cells with low density were spotted from samples immediately after seeding (Fig. 3A, B) and with 180 minutes incubation time (Fig. 3I, J). Majority of the sample was found with no cell attached. Much more densely attached cells were observed on samples with 45 (Fig. 3C, D) and 90 minutes (Fig. 3E, F) incubation time after seeding. In some parts of the sample, almost confluent monolayers were formed. Samples with 135 minutes incubation time (Fig. 3E, F) exhibited moderate cell attachment, which also agreed with the AlamarBlue ${ }^{\mathrm{TM}}$ results.
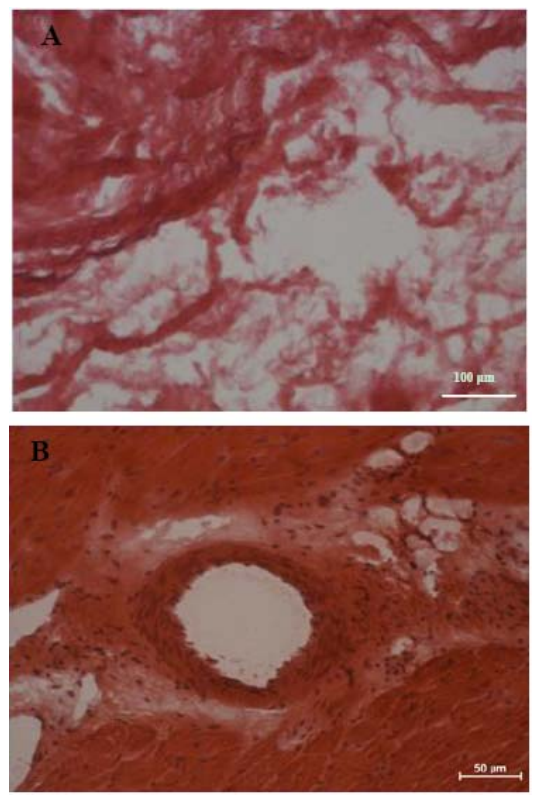

Fig. 2. H\&E staining for acellular ECM (A) and native tissue (B).
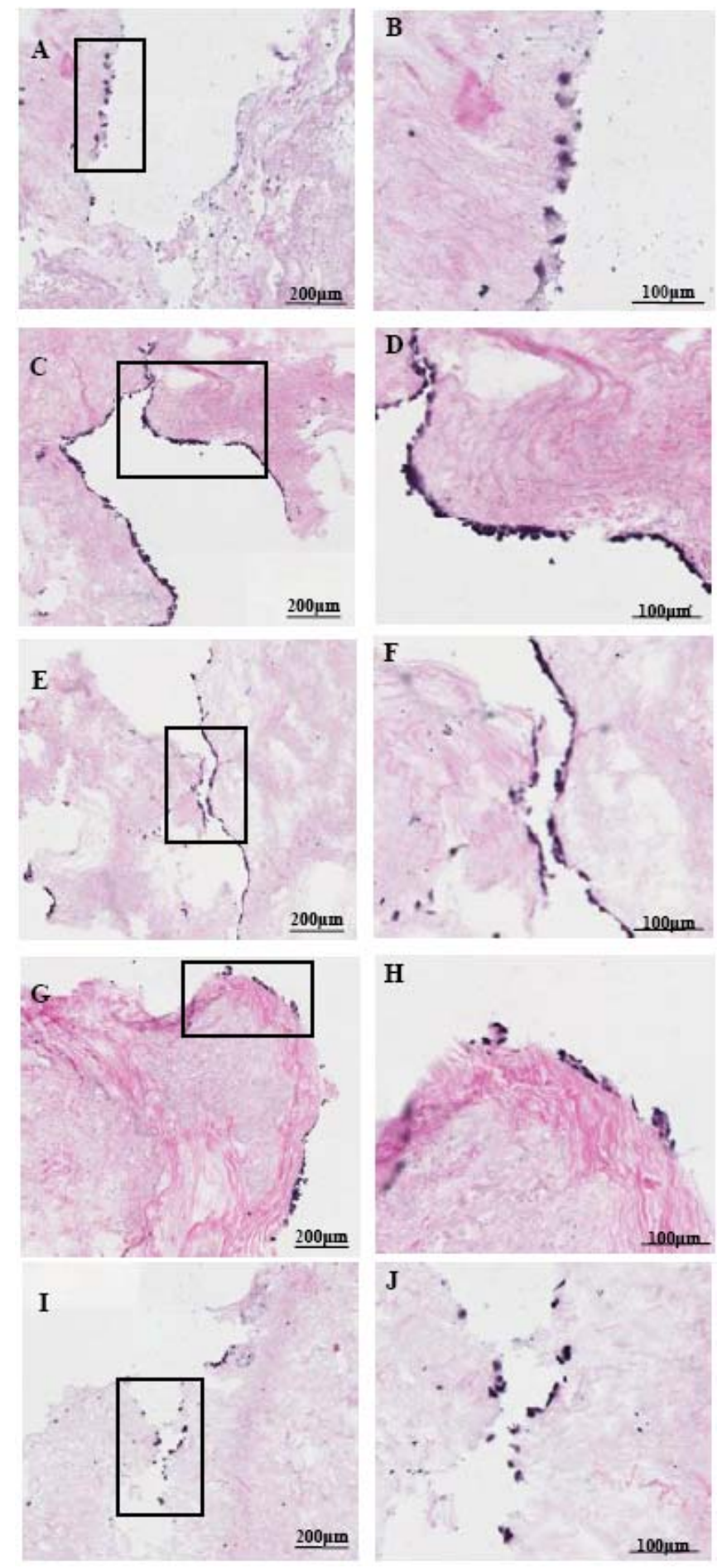

Fig. 3. H\&E images for HUVEC attachment to ECM utilizing various attachment times, after static incubation for 0 minutes (A: $4 x, \mathrm{~B}: 10 x), 45$ minutes $(\mathrm{C}: 4 x, \mathrm{D}: 10 x), 90$ minutes $(\mathrm{E}: 4 x, \mathrm{~F}: 10 x), 135$ minutes $(\mathrm{G}: 4 x, \mathrm{H}$ : $10 x$ ), and 180 minutes (I: $4 x, \mathrm{~J}: 10 x$ ).

\section{B. Cell Morphology}

Confocal microscopy imaging showed that HUVECs attached and aligned 7 days after seeding (Fig. 4A) while MSCs were relatively less arranged but more densely packed against each other (Fig. 4B). When co-cultured together (Fig. 4C), DiI-labelled HUVECs were seen even more uniformly arranged, better spread and aligned than singly cultured ones. 

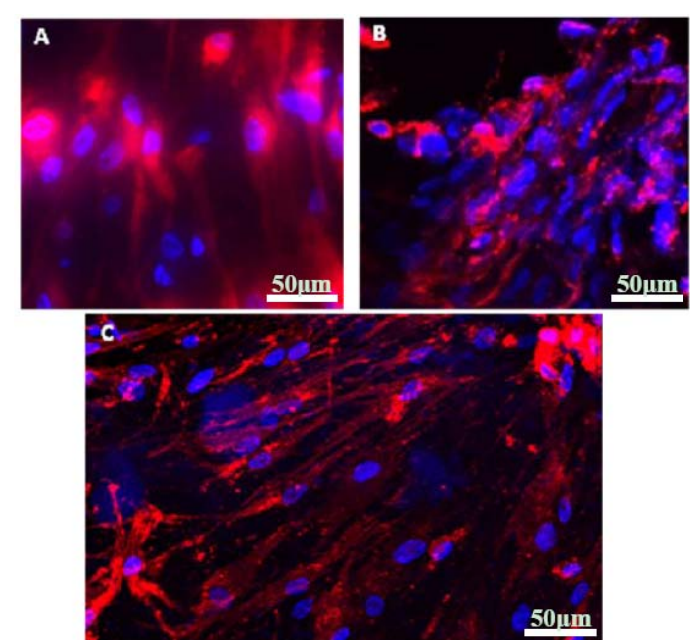

Fig. 4. Rearrangement of HUVEC and MSC reseeded on ECM. Confocal images of HUVEC (A), MSC (B), and simultaneous co-culture at 1:1 ratio (C) cultured for 7 days. Cells were pre-labeled (prior to seeding) with DiI (red) and counterstained with DAPI (blue) for cell nuclei. In the co-culture, only the HUVECs were pre-labeled with DiI.

The cell morphology on the ECM surface was further analyzed by scanning electron microscopy. Figure 5B shows co-cultured HUVECs and MSCs densely attached to the ECM surface in contrast to acellular ECM (Fig. 5A). A certain degree of cell alignment can be seen and cell-cell interactions were found presumably through gap junctions. "Bridge" formed by cells were found across the gap on the surface of ECM.

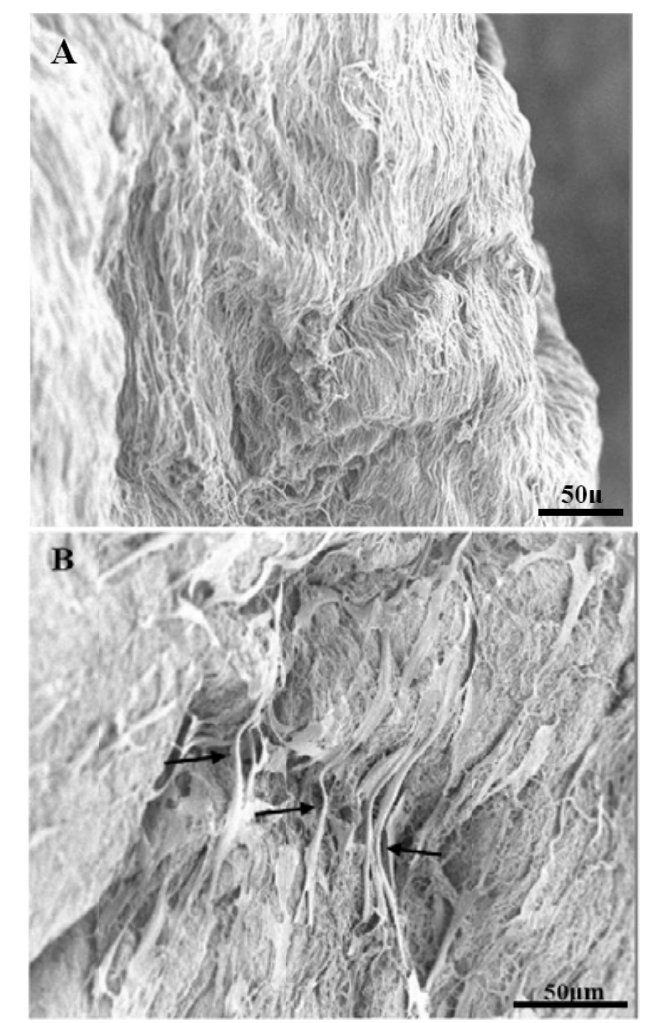

Fig. 5. SEM image of acellular ECM (A) and HUVEC-seeded ECM (B) surface. Arrows indicate "bridges" formed by cells across the gap on the scaffold surface.

\section{Cell Proliferation}

The proliferation of HUVEC and MSC reseeded on ECM treated with laminin, fibronectin, and gelatin was measured using the AlamarBlue ${ }^{\mathrm{TM}}$ assay. Significant improvement in HUVEC growth was observed on all treated ECM samples compared to the untreated control, especially the ones treated with fibronectin and gelatin (Fig. 6A, $p<0.001$ ). MSCs alone exhibited better overall proliferation only on the fibronectin-treated scaffold, which was statistically significant over the 21 days culture (Fig. 6B), while the proliferation of MSC-HUVEC co-cultures, similarly to HUVEC alone, was best on fibronectin- and gelatin-coated ECM (Fig. 6C). Sequential co-culture also led to improved cell attachment and growth compared to simultaneous co-culture and single culture (unpublished data). However, no additional improvement was observed with ECM treatment (Fib. 6D).
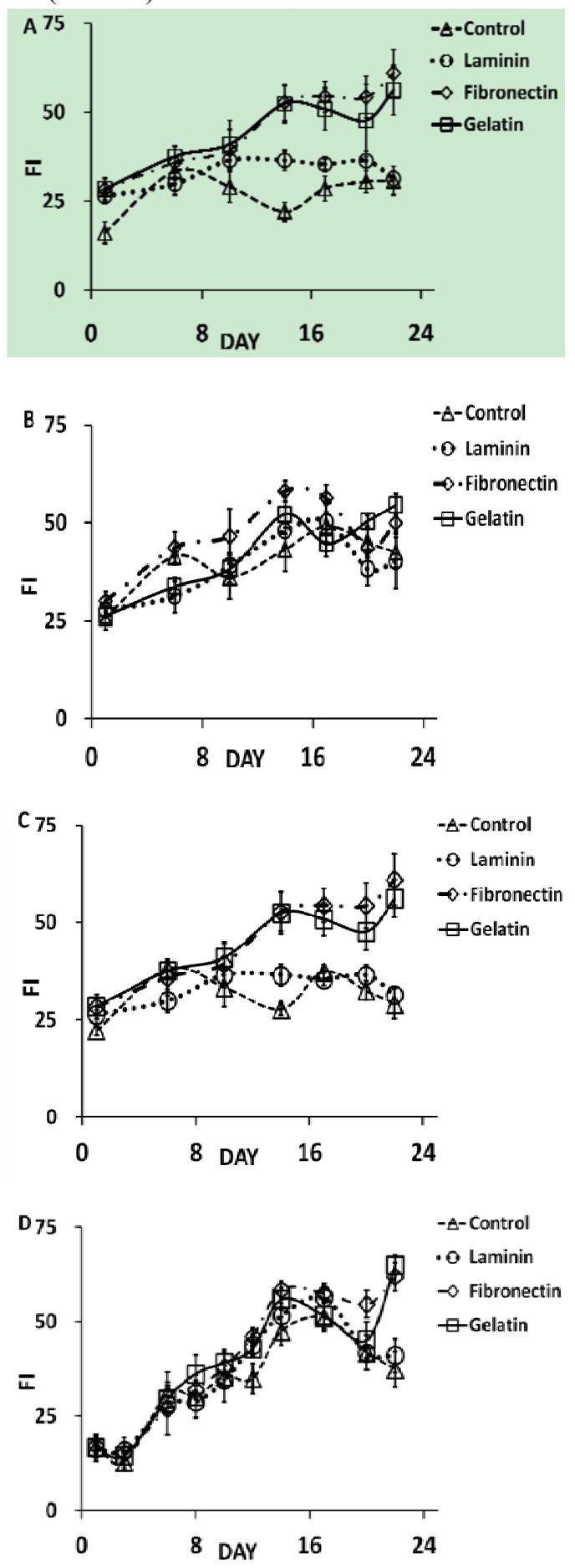

Fig. 6. Cell proliferation for 21 days on biochemically modified ECM scaffolds. HUVEC (A), MSC (B), simultaneous co-culture (C), and sequential co-culture (D) on untreated ECM pieces (control) and ECM treated with laminin, fibronectin, and gelatin. Cell viability is presented as AlamarBlue ${ }^{\mathrm{TM}}$ Fluorescent intensity (FI). 


\section{Discussion}

Certain parameters, such as the attachment time and scaffold surface properties, need to be optimized to achieve uniform attachment and growth of endothelial cells on acellular ECM scaffolds. For practical reasons and to allow efficient and broad screening of a variety of factors, the cells in this case were seeded onto the surface of tissue that was shown to resemble the ECM vasculature. A small seeding volume of $40 \mu \mathrm{l}$ was used to confine the seeding area and prevent cell suspension overflow from the surface of ECM slices. The optimal incubation time to allow cell attachment was found to be crucial. On the one hand, too short the period is not sufficient for cells to attach to the ECM scaffold and may result in cells being washed away during subsequent medium replacement. Too long an incubation time, on the other hand, can compromise cell viability presumably due to complete nutrient consumption in the limited media volume available, resulting in cell detachment and death. Results generated from both the AlamarBlue ${ }^{\mathrm{TM}}$ assay and the H\&E staining were found to be consistent with each other, implying that an incubation time of 45 to 90 minutes, after seeding, should be applied. Healthy and functional blood vessel should be covered by a complete confluent mono-layer of endothelial cells [14], [15]. The cell layer after 45 minutes incubation time (Fig. 3C, D) appeared similar to vasculature, which is outlined by multilayer of presumably smooth muscle cells in healthy native tissue (Fig. 2B) implying that acellular ECM can serve as an excellent candidate for re-endothelialization of regenerated cardiac tissue.

Multipotent MSCs, harboring therapeutic potential, were shown to support the attachment of endothelial cells and enhance angiogenesis [16], making them ideal candidates for applications involving cardiovascular tissue regeneration. Many studies have verified the ability of MSCs in supporting the long-term survival and stability of endothelial cells [16], [17] when they are co-cultured in the same niche. Stable and functional blood vessel network can be regenerated in vivo by injecting mixed mesenchymal precursor cells and HUVECs, whereas tubular network formed by HUVECs alone tend to regress and disappear over time [12]. Our previous studies demonstrated that when being co-cultured on tissue culture plate, MSCs and HUVECs exhibited some mutual effects, which influence the overall population dynamics and growth over time (results not shown). Here, confocal and SEM imaging showed that MSCs affected the alignment and morphology of HUVECs on ECM, which could result from physical contact or signaling proteins secreted from the cells.

Our results indicated that the sequence of seeding is another essential factor influencing the endothelial cell proliferation. In a different study, we demonstrated that MSCs enhanced the proliferation of HUVECs on 3-dimensional ECM scaffold using only sequential co-culture method (unpublished data). Simultaneous co-culture approach was applied in mice models because the differentiated mural precursor cells expressing pericyte markers can efficiently recruit mural cells from the surrounding native tissue, providing endothelial-mural cell interaction, which is critical for long-term survival and stability of endothelial cells [13]. In our acellular ECM model, under in vitro condition, no surrounding mural cells are available for recruitment; hence pre-formed layer of MSCs or MSC-derived supporting cells is needed with sequential seeding approach

Chemical modification using various proteins to coat the ECM revealed that one or two types of proteins are required for the attachment and proliferation of each cell type. Even though characterization of thin acellular ECM from our previous studies demonstrated that after decellularization, collagen type I, III, and fibronectin, which are essential proteins for cell attachment were preserved with high quantities comparable to native tissue [7], the relatively low attachment efficiency and proliferation of HUVECs indicated that additional protein molecules were required to enhance cell attachment and proliferation on thick decellularized ECM scaffold. It is also a common practice to coat tissue culture plate with gelatin prior to HUVECs plating to enhance their attachment and growth. ECM coated with gelatin or fibronectin exhibited enhanced cell growth for HUVECs alone and simultaneous co-cultured cells. Sequential approach with MSC seeding on ECM prior to HUVECs might modify the local niche and prepare the ECM scaffold for HUVEC attachment. However, additional chemical treatment did not further enhance the advantage of sequential seeding over single culture approach, indicating that preparing ECM by pre-seeding with MSCs and by coating with protein solutions might serve the same function in preparing the ECM for HUVECs. On the other hand, simultaneous co-culture approach, which did not exhibit any increase in cell growth with non-treated or laminin-treated ECM scaffold, showed significant improvement with gelatin and fibronectin treatment. This improvement in performance resembled strikingly to those seeded with HUVECs alone, implying that chemical treatment, instead of co-culture, in this case is the dominant factor for enhanced cell proliferation. Unlike HUVECs, MSCs showed fluctuating but prolonged proliferation over the three weeks culture with or without chemical treatment. Out of the three protein types, only fibronectin improved MSCs growth with statistical significance as indicated by ANOVA analysis. Fibronectin coating that led to significantly improved growth of both singly and co-cultured cells may be the best choice among the three.

\section{CONCLUSION}

Results from this study validated the advantages of co-culturing MSCs and HUVECs and the potential of acellular ECM to be used as scaffold material for cardiovascular tissue engineering. With biochemical modification, thick ECM can support the growth and stabilization of endothelial cells over time - the most important requirement for vascularization and angiogenesis. Effective regeneration of thick tissue with functional blood vessels also requires supportive environment for sufficient oxygen and nutrients supply. Dynamic culturing with bioreactors can provide recellularized tissue with nutrient and waste exchange, physiological environment mimicking their natural condition, and is currently being studied by our group. With the combination of precise cell selection, supporting scaffold, and accommodating culture environment, re-generated thick cardiac tissue with functional blood 
vessels can serve as an optimal solution for partial cardiac replacement therapy.

\section{ACKNOWLEDGEMENTS}

This research is supported by the Singapore National Research Foundation under the CREATE program: The Regenerative Medicine Initiative in Cardiac Restoration Therapy Research Program; and The Israeli Science Foundation (ISF) under grant no.: 1563/10 entitled: "Thick acellular heart ECM with inherent vasculature: Towards engineering cardiac tissue replacement".

\section{REFERENCES}

[1] Q. Chen, S. Harding, N. Ali, A. Lyon, and A. Boccaccini, "Biomaterials in cardiac tissue engineering: Ten years of research survey," Mat Sci Eng R, vol. 59, no. 1-6, pp. 1-37, February 2008.

[2] H. Jawad, N. Ali, A. Lyon, Q. Chen, S. Harding, and A. Boccaccini, "Myocardial tissue engineering: A review," J Tissue Eng Regen Med, vol. 1 , no. 5, pp. 327-342, November 2007.

[3] C. L. Visage, B. Dunham, P. Flint, and K. W. Leong, "Coculture of mesenchymal stem cells and respiratory epithelial cells to engineer a human composite respiratory mucosa," Tissue Eng, vol. 10, pp. 1426-1435, September 2004.

[4] S. Levenberg, J. Rouwkema, M. Macdonald, E. S. Garfein, D. S. Kohane, D. C. Darland, R. Marini, C. A. van Blitterswijk, R. C Mulligan, P. A. D'Amore, and R. Langer, "Engineering vascularized skeletal muscle tissue," Nat Biotechnol, vol. 23, pp. 879-884, June 2005.

[5] S. G. Ball, A. C. Shuttleworth, and C. M. Kielty, "Direct cell contact influences bone marrow mesenchymal stem cell fate," Int J Biochem Cell Biol, vol. 36, pp. 714-727, April 2004.

[6] M. Radisic and G. Vunjak-Novakovic, "Cardiac tissue engineering," $J$ Serb Chem Soc, vol. 70, 541-556, 2005.

[7] Y. Eitan, U. Sarig, N. Dahan, and M. Machluf, "Acellular cardiac extracellular matrix as a scaffold for tissue engineering: In vitro cell support, remodeling, and biocompatibility," Tissue Eng: Part C Methods, vol. 16, pp. 671-683, August 2010.

[8] H. C. Ott, T. S. Matthiesen, S. K. Goh, L. D. Black, S. M. Kren, and T. I. Netoff, and D. A. Taylor, "Perfusion-decellularized matrix: using nature's platform to engineer a bioartificial heart," Nat Med, vol. 14, pp. 213-221, January 2008.

[9] U. Sarig, G. Au-Yeung, Y. Wang, T. Bronshtein, N. Dahan, Y. C. Boey, S. S. Venkatraman, and M. Machluf, "Thick acellular heart extracellular matrix with inherent vasculature: Potential platform for myocardial tissue regeneration," Tissue Eng: Part A, vol. 18, July 2012.

[10] T. Kaully, K. K. Francis, A. Lesman, S. Levenberg, "Vascularization-the conduit to viable engineered tissues," Tissue Eng Part B Rev, vol. 15, pp. 159-169, June 2009.

[11] R. K. Jain, "Molecular regulation of vessel maturation," Nat Med, vol. 9, pp. 685-693, June 2003.

[12] N. Koike, D. Fukumura, O. Gralla, P. Au, J. S. Schechner, and R. K. Jain, "Tissue engineering: creation of long-lasting blood vessels," Nature, vol. 428, pp. 138-139, March 2004.

[13] K. K. Hirschi, S. A. Rohovsky, and P. A. D'Amore, "PDGF, TGF-beta, and heterotypic cell-cell interactions mediate endothelial cell-induced recruitment of $10 \mathrm{~T} 1 / 2$ cells and their differentiation to a smooth muscle fate," J Cell Biol, vol. 141, pp. 805-814, May 1998.

[14] M. Kolbe, Z. Xiang, E. Dohle, M. Tonak, C. J. Kirkpatrick, and S. Fuchs, "Paracrine effects influenced by cell culture medium and consequences on microvessel-like structures in cocultures of mesenchymal stem cells and outgrowth endothelial cells," Tissue Eng Part A, vol. 17, pp. 2199-2212, September 2011.

[15] J. A. Cook and J. B. Mitchell, "Viability measurements in mammalian cell systems," Anal Biochem, vol. 179, pp. 1-7, May 1989.

[16] J. M. Sorrell, M. A. Baber, and A. I. Caplan, "Influence of adult mesenchymal stem cells on in vitro vascular formation," Tissue Eng Part A, vol. 15, pp. 1751-1761, July 2009.

[17] P. Au, L. M. Daheron, D. G. Duda, K. S. Cohen, J. A. Tyrrell, R. M. Lanning, D. Fukumura, D. T. Scadden, and R. K. Jain, "Differential in vivo potential of endothelial progenitor cells from human umbilical cord blood and adult peripheral blood to form functional long-lasting vessels," Blood, vol. 111, pp. 1302-1305, February 2008. 